\title{
Combining CSP and Object-Z: Finite or Infinite Trace Semantics?
}

\author{
Clemens Fischer \\ Universität Oldenburg, FB Informatik, Abt. Semantik,PO Box 2503, \\ 26129 Oldenburg, Germany, fischer@informatik.uni-oldenburg.de
}

\section{Graeme Smith}

Technische Universität Berlin, FB Informatik, Sekr. FR 5-6, Franklinstr.28/29, 10587 Berlin, Germany,graeme@cs.tu-berlin.de

\begin{abstract}
In this paper we compare and contrast two alternative semantics as a means of combining CSP with Object-Z. The purpose of this combination is to more effectively specify complex, concurrent systems: while CSP is ideal for modelling systems of concurrent processes, Object- $\mathrm{Z}$ is more suitable for modelling the data structures often needed to model the processes themselves. The first semantics, the finite trace model, is compatible with the standard CSP semantics but does not allow all forms of unbounded nondeterminism to be modelled (i. e. where a choice is made from an infinite set of options). The second semantics, the infinite trace model, overcomes this limitation but is no longer compatible with the standard CSP semantics. Issues involving specification, refinement and modelling fairness are discussed.
\end{abstract}

\section{Keywords}

CSP, Object-Z, concurrent systems, combining FDTs, semantics, refinement

\section{INTRODUCTION}

CSP [15] is a process algebra developed for the formal specification of concurrent systems. The standard semantics of CSP is the failures-divergences semantics [3] where finite traces are used together with refusal sets of events to model a process' external behaviour. This semantics, however, does not support unbounded nondeterminism (where a process can choose from an infinite set of options) thus restricting what can be expressed in the language. For example, it is not possible to specify a process which nondeterministically chooses, and then outputs, any natural number.

To overcome this limitation, two "alternative" semantics of CSP have been proposed. The first [18], which we will refer to as the finite trace model, doesn't alter the 
form of the semantics, i.e. finite traces and refusal sets are used, nor the meaning of the CSP operators, but provides an alternative way of defining these operators which allows some forms of unbounded nondeterminism to be modelled. For example, a process which nondeterministically selects any natural number, outputs this natural number and then stops can be modelled in this semantics. It does not, however, allow the specification of a process which nondeterministically selects any natural number $n$ and then performs a particular event $n$ times. More precisely, because it only uses finite traces to model a process, it cannot distinguish between a process which can undergo any finite sequence of an event $a$ and a process which can also undergo an infinite sequence of $a$ 's. The second semantics $[20,19]$, which we will refer to as the infinite trace model, overcomes this problem by introducing the set of infinite traces of the process. It can model all forms of unbounded nondeterminism but its form, and the meaning of some operators, are different from those of the standard failuresdivergences semantics.

These alternative semantics of CSP allow it to be combined with state-based specification languages where unbounded nondeterminism arises naturally. For example, the finite trace model has been used as a common semantics for Z [24] and CSP in [10], and Object-Z [9] and CSP in [23] and [11]. The purpose of such combinations is to more effectively specify complex, concurrent systems: the state-based language is used for the specification of data structures for which CSP is not particularly suited. This need for more than one specification language to model complex, concurrent systems has also arisen in the ODP (Open Distributed Processing) standardisation initiative [16] where a combination of $Z$ and LOTOS [2] has been proposed [7].

In this paper, we compare and contrast the two alternative semantics as a means of integrating CSP with Object-Z. The advantage of using Object- $Z$ over $Z$ is that it is object-oriented and therefore has a class structure which encapsulates state and operations. Such a structure is readily identifiable with a process as illustrated in [22]. The lack of a similar structure in $\mathrm{Z}$ makes such an integration more difficult. Indeed, [7] requires an intermediate language ZEST [5] which also has a class structure and is (automatically) translatable to $Z$, in order to define a common semantics with the process algebra part of LOTOS. Also, [10] proposes additional syntax for grouping the $\mathrm{Z}$ schemas corresponding to a process.

In Section 2, we provide a brief introduction to Object- $Z$ and, in Section 3, we define the alternative CSP semantics. Section 4 then looks at integrating Object-Z and CSP using each of the semantics. A case study illustrating the difference between the two approaches is presented in Section 5 and rules of refinement in Section 6. In Section 7, we look at how the infinite trace semantics can be used to specify fairness properties.

\section{OBJECT-Z}

Object-Z [9] is an extension of Z [24] designed to support an object-oriented specification style. It includes a special class construct to encapsulate a state schema with all the operations which may affect its variables. A class is represented syntactically by a named box possibly with generic parameters. In this box there may be local type and constant definitions, at most one state schema and associated initial state schema, and 
zero or more operation schemas. As an example, consider the following specification of a bounded buffer. This specification is generic since the type $T$ of the items in the buffer is not specified.

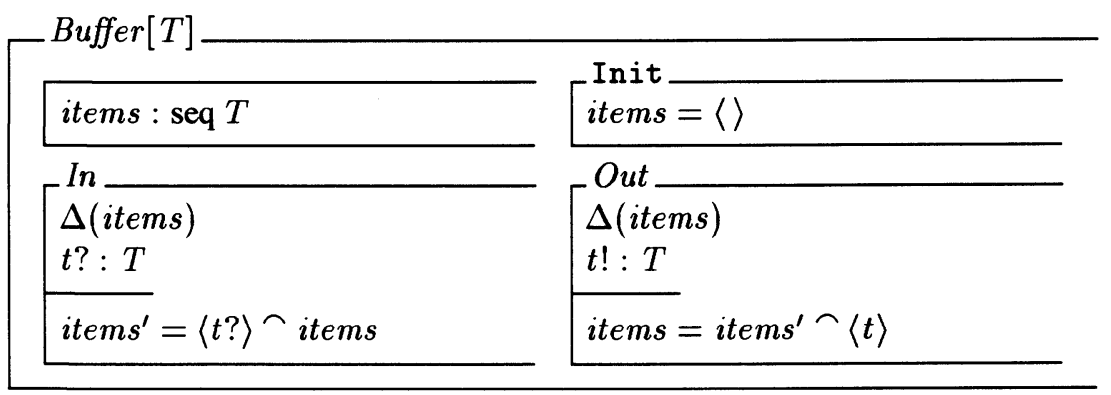

The class has a single state variable items denoting the items in the buffer. Initially, the buffer is empty and the operations In and Out enable items to be input to and output from the buffer, respectively, on a first-in/first-out basis. Each operation schema has a $\Delta$-list of state variables which it may change, a declaration part consisting of input (denoted by names ending in ?) and output (denoted by names ending in !) parameters and a predicate part relating the pre- and post-values (denoted by names ending in ') of the state variables.

In addition, a class may have a history predicate enabling the specification of fairness properties. This will be discussed further in Section 7 .

\section{MODELLING UNBOUNDED NONDETERMINISM IN CSP}

In this section, we present the details of the alternative semantics of CSP which enable the modelling of unbounded nondeterminism. We let $a$ denote events, $X$ and $Y$ denote sets of events, $s$ and $t$ denote finite traces and $u$ denote infinite traces.

The finite trace model of CSP is presented in [18]. As in the standard failuresdivergences semantics [3], a process is modelled by its failures $\mathcal{F}$ and divergences $\mathcal{D}$. The failures of a process are pairs $(s, X)$ where $s$ is a finite trace that the process may undergo and $X$ is a set of events that the process may refuse to perform after undergoing $s$. That is, if the process after undergoing $s$ is in an environment which only allows it to undergo events in $X$, it may deadlock. The divergences of a process are the traces after which the process may undergo an infinite sequence of internal events, i.e. livelock. Failures and divergence are defined in terms of a non-empty alphabet of events $\Sigma$ as follows.

Failures: $\mathcal{F} \subseteq \Sigma^{*} \times \mathbb{P} \Sigma$ such that $\mathcal{F}$ is not empty and the following axioms hold.

(1) $\quad(s \frown t, \varnothing) \in \mathcal{F} \Rightarrow(s, \varnothing) \in \mathcal{F}$

(2) $(t, X) \in \mathcal{F} \wedge Y \subseteq X \Rightarrow(t, Y) \in \mathcal{F}$

(3) $(t, X) \in \mathcal{F} \wedge \forall a \in Y \bullet(t \frown\langle a\rangle, \varnothing) \notin \mathcal{F} \Rightarrow(t, X \cup Y) \in \mathcal{F}$

Axiom 1 captures the requirement that the traces of a process are prefix-closed. Axiom 2 states that if a process can refuse all events in a set $X$ then it can refuse all 
events in any subset of $X$. Axiom 3 states that a process can refuse any event which cannot occur as the next event.

In addition, the standard failures-divergences semantics [3] has an axiom which states that all events in a set can be refused if all events in its finite subsets can be refused. Hence, a process which selects, and then outputs, any natural number cannot be modelled. Such a process would be able to refuse to output all numbers in any finite set of natural numbers, but not all numbers in the set of all natural numbers.

This axiom was included in the failures-divergence semantics to make the nondeterminism order on processes complete which was necessary to define the fixed point theory. However, the finite trace model, by defining the same fixed point theory in a different way (see [19] for details), removes the need for this axiom and, hence, removes the associated restriction on modelling unbounded nondeterminism.

Divergences: $\mathcal{D} \subseteq \Sigma^{*}$ such that the following axioms hold.

(4) $s \in \mathcal{D} \Rightarrow s^{\curvearrowleft} t \in \mathcal{D}$

(5) $s \in \mathcal{D} \Rightarrow(s \frown t, X) \in \mathcal{F}$

These axioms capture the idea that it is impossible to determine anything about a divergent process in a finite time. Therefore, the possibility that it might undergo further events cannot be ruled out. In other words, a divergent process behaves chaotically.

The infinite trace model, presented in $[20,19]$, is an extension of the finite trace model which allows all forms of unbounded nondeterminism to be modelled. In particular, it can distinguish between a process which can undergo any finite sequence of an event $a$ and one which can also undergo an infinite sequence of $a$ 's.

In addition to failures and divergences, it also includes a component $\mathcal{I}$ corresponding to the infinite traces of a class. $\mathcal{I}$ is defined in terms of the alphabet of events $\Sigma$ as follows.

Infinite traces: $\mathcal{I} \subseteq \Sigma^{\omega}$ such that the following properties hold*

(6) $s \frown u \in \mathcal{I} \Rightarrow(s, \varnothing) \in \mathcal{F}$

(7) $s \in \mathcal{D} \Rightarrow s \wedge u \in \mathcal{I}$

(8) $(s, \varnothing) \in \mathcal{F} \Rightarrow$

$\exists T \bullet \forall t \in T \bullet\left(s^{\frown} t,\left\{a \mid t^{\frown}\langle a\rangle \notin T\right\}\right) \in \mathcal{F} \wedge\left\{s^{\frown} u \mid u \in \bar{T}\right\} \subseteq \mathcal{I}$

where $\Sigma^{\omega}$ is the set of infinite traces over $\Sigma$ and $T$ is a non-empty, prefix-closed set of finite traces and $\bar{T}=\left\{u \in \Sigma^{\omega} \mid \forall t<u \bullet t \in T\right\}$.

Axioms 6 and 7 are straightforward extensions of axioms 1 and 4 respectively. The purpose of axiom 8 is to ensure there are enough infinite traces analogously to the way that axiom 3 ensures there are enough failures. It states that there exists at least one deterministic refinement of a process after it has undergone a trace $s$ (the finite traces of this refinement are given by the set $T$ ), and that any infinite trace that this refinement can undergo is in $\mathcal{I}$. The explanation and derivation for this axiom are quite subtle and the interested reader is referred to [19] for details.

*We adopt the form of axiom 8 from [20] which is equivalent to that in [19] as argued in the appendix of that paper. 


\section{MODELLING OBJECT-Z CLASSES AS PROCESSES}

In this section we present both finite and infinite trace models for Object- $\mathrm{Z}$ classes. The former is based on the approaches adopted in [23] and [11]. The latter is a simple extension of these approaches and proofs of the necessary additional axioms are given. As a preliminary, we present the semantics of Object- $Z$ classes from which the finite and infinite trace models are derived.

\subsection{Semantics of Object-Z classes}

A class in Object- $Z$ can be modelled as a set of values each corresponding to a potential object of the class at some stage of its evolution. Such a semantics is presented in [22] where the value chosen to represent an object is the sequence of states the object has passed through together with the corresponding sequence of operations the object has undergone. This value is referred to as the history of the object.

The histories of a class can be derived from its sets of states $S$, initial states $I$, operations $O$ and state transitions $S T$. Object-Z classes can also have fairness properties which restrict the set of histories. These, however, are not considered in this section.

The states of a class are an assignment of values to the class' local constants and state variables as well as any global constants to which the class may refer. Given the set of all possible identifiers $I d$ and the set of all possible values Value, the states of a class can be represented by a set $S \subseteq(I d \cdots$ Value $)$ such that the states of a class refer to a common set of identifiers: $s_{1} \in S \wedge s_{2} \in S \Rightarrow \operatorname{dom} s_{1}=\operatorname{dom} s_{2}$

The initial states of a class are simply a subset of its states: $I \subseteq S$. The operations of a class are instances of the class' operation schemas. They can be represented by the name of an operation schema together with an assignment of values to its parameters. The operations of a class can be represented by a set $O \subseteq I d \times(I d \#$ Value $)$ such that an operation name is always associated with the same set of parameters: $\left(n, p_{1}\right) \in O \wedge\left(n, p_{2}\right) \in O \Rightarrow \operatorname{dom} p_{1}=\operatorname{dom} p_{2}$

Finally, the state transitions are represented by an operation together with a pair of states representing a possible pre-state and corresponding post-state of the operation: $S T \subseteq O \times S \times S$.

No conditions are placed on the state transitions allowing an operation to be associated with no transitions (corresponding to having an unsatisfiable precondition) or multiple transitions with the same pre-state (corresponding to nondeterminism).

A history of a class is a non-empty sequence of states together with a sequence of operations. Either both sequences are infinite or the state sequence is one longer than the operation sequence. The first state is an initial state of the class and each pair of consecutive states corresponds to a state transition of the corresponding operation.

Given a class $C$ with states $S$, initial states $I$, operations $O$ and state transitions $S T$, the histories of the class are derived as follows.

$$
\begin{gathered}
\mathcal{H}(C)=\left\{(s, o) \mid\left(s \in S^{\omega} \wedge o \in O^{\omega} \vee s \in S^{*} \wedge o \in O^{*} \wedge \# s=\# o+1\right) \wedge\right. \\
s(1) \in I \wedge \forall i \in \operatorname{dom} o \bullet(o(i), s(i), s(i+1)) \in S T\}
\end{gathered}
$$

Note that the interpretation of an operation in Object- $Z$ differs from that in $Z$ in that an 
operation cannot occur when its precondition is not enabled. In $\mathrm{Z}$, the operation would be able to occur but the outcome would be unspecified. In [11], a slightly different interpretation of an operations is taken allowing both the views of Object- $\mathrm{Z}$ and $\mathrm{Z}$ to be accommodated. In this paper, however, we adopt the standard view of Object-Z.

The following property of the set of histories of a class (without fairness properties) is given as a lemma without proof.

Lemma 1: An infinite history $(s, o)$ is a history of $C$ if and only if all its prefixes are histories of $C$ :

$$
\begin{aligned}
& \forall(s, o) \in S^{\omega} \times O^{\omega} \bullet(s, o) \in \mathcal{H}(C) \Leftrightarrow \\
& \forall n \in \mathbb{N} \bullet(1 \ldots n+1 \triangleleft s, 1 \ldots n \triangleleft o) \in \mathcal{H}(C)
\end{aligned}
$$

\subsection{A finite trace model of Object-Z classes}

In order to model a class as a process, we need to relate operations and events. A number of ways of doing this exist (see [23] and [11] for examples). For the purposes of this paper, we will assume the existence of a function event which returns a unique event for each operation of a class. This function also returns identical events for operations from different classes which have the same name and whose parameters have the same basenames (i.e. apart from the? or!) and values. This allows such commonnamed operations to synchronise and their matching input and output parameters to be equated. The function events is the straight-forward extension of event to traces. A class which has unsatisfiable initial conditions will have no histories and hence cannot be given a failures-divergences semantics in an obvious way. Such classes need to be considered separately from other classes.

The failures of a class $C$ with satisfiable initial conditions, and hence at least one history, can be derived from its set of histories as follows: $(t, X)$ is a failure of $C$ if there exists a history of $C$ such that

- the sequence of operations of the history corresponds to the sequence of events in $t$ and

- for each event in $X$, there does not exist a history which extends the original history by an operation corresponding to that event.

$$
\begin{gathered}
\mathcal{F}(C)=\{(t, X) \mid \exists(s, o) \in \mathcal{H}(C) \bullet t=\operatorname{events}(o) \wedge \\
\forall e \in X \bullet \nexists s t \in S, o p \in O \bullet \\
\quad e=\operatorname{event}(o p) \wedge(s \frown\langle s t\rangle, o \frown\langle o p\rangle) \in \mathcal{H}(C)\}
\end{gathered}
$$

As shown in [23], the failures of $C$ defined in this way satisfy axioms 1 to 3 of the finite trace model.

Since Object-Z has no notion of internal operations nor recursive definitions of operations*, the set of divergences of a class $C$ is empty: $\mathcal{D}(C)=\varnothing$. This trivially satisfies the properties 4 and 5 of the finite trace model.

\footnotetext{
*Although recursive definitions of operations have been suggested for Object-Z (e.g. [8]), we have adopted a more conservative view of Object-Z in this paper.
} 


\subsection{An infinite trace model of Object- $Z$ classes}

The infinite trace model can be derived from the finite trace model by the addition of a set of infinite traces. Given a class $C$ with operations $O$ these are derived from the histories of $C$ as follows.

$$
\mathcal{I}(C)=\left\{t \mid \exists(s, o) \in \mathcal{H}(C) \bullet o \in O^{\omega} \wedge t=\text { events }(o)\right\}
$$

The proof of axiom 6 of the infinite trace model follows from Lemma 1. The proof of axiom 7 is trivial since the set of divergences of a class is empty. It remains then to prove axiom 8 .

Let $C$ be a class with states $S$, initial states $I$, operations $O$ and state transitions $S T$ and let $(t, \varnothing)$ be a failure of $C$. From the definition of the function $\mathcal{F}$, there exists a history $(s, o)$ of $C$ such that $t=$ events $(o)$.

The proof of axiom 8 relies on finding another class $D$ whose initial state is the final state of $s$ and is otherwise a deterministic refinement of class $C$. Such a class can be constructed as follows.

Let $D$ be a class with states $S$, initial states $I^{\prime}=\{\operatorname{last}(s)\}$, operations $O$ and state transitions $S T^{\prime} \subseteq S T$ such that

$$
\begin{aligned}
& \forall o p \in O, s t_{1} \in S \bullet \exists s t_{2} \in S \bullet \\
& \quad\left(o p, s t_{1}, s t_{2}\right) \in S T \Rightarrow \exists_{1} s t \in S \bullet\left(o p, s t_{1}, s t\right) \in S T^{\prime}
\end{aligned}
$$

That is, whenever $C$ can perform an operation from a particular state, $D$ can also perform the operation from that state and there is a unique post-state.

The set $T$ whose existence must be found to satisfy axiom 8 is then simply the set of traces of $D$, i.e. $T=\{t \mid(t, X) \in \mathcal{F}(D)\}$. To prove this, we need to prove the following for $T$.

(1) $T$ is prefix-closed and non-empty.

(2) $\forall t \in T \bullet(s \frown t,\{a \mid t \frown\langle a\rangle \in T\}) \in \mathcal{F}(C)$.

(3) $\{s \frown u \mid u \in \bar{T}\} \subseteq \mathcal{I}(C)$ where $\bar{T}=\left\{u \in \operatorname{events}\left(O^{\omega}\right) \mid \forall t<u \bullet t \in T\right\}$.

\section{Proof}

(1) The failures of a class are prefix-closed and non-empty.

(2) By axiom 2 of the finite trace model, $\forall t \in T \bullet(t, \varnothing) \in \mathcal{F}(D)$. Hence, by axiom 3 of the finite trace model, $\forall t \in T \bullet(t,\{a \mid(t \frown\langle a\rangle, \varnothing) \notin \mathcal{F}(D)\}) \in$ $\mathcal{F}(D)$ and, therefore, $\forall t \in T \bullet(t,\{a \mid t \frown\langle a\rangle \notin T\} \in \mathcal{F}(D)$. Also, from the definition of $D$, it can be shown that $\{(s \frown t, X) \mid(t, X) \in \mathcal{F}(D)\} \subseteq \mathcal{F}(C)$. Therefore, $\forall t \in T \bullet\left(s \frown t,\left\{a \mid t^{\frown}\langle a\rangle \notin T\right\}\right) \in \mathcal{F}(C)$.

(3) Given $u \in \bar{T}$, let $t_{n}=1 \ldots n \triangleleft u$ for all $n \in \mathbb{N}$. Since for all $n \in \mathbb{N}, t_{n} \in T$, from the definition of $\mathcal{F}$, there exists a history $\left(s_{n}, o_{n}\right)$ of $D$ such that $t_{n}=\operatorname{events}\left(o_{n}\right)$. Since $D$ is deterministic, each sequence of operations will only occur with one sequence of states in the set of histories of $D$. Also, since event returns a unique event for each operation of a class, any sequence of events of $D$ will correspond to a unique sequence of operations and hence a unique sequence of states. Therefore, $\forall m, n \in \mathbb{N} \bullet m<n \Rightarrow s_{m}<s_{n}$. 
Let $(s, o)$ be the infinite history such that $\forall n \in \mathbb{N} \bullet s(n)=\operatorname{last}\left(s_{n}\right)$ and $o=$ events $(u)$. Then $\forall n \in \mathbb{N} \bullet(1 \ldots n+1 \triangleleft s, 1 \ldots n \triangleleft o) \in \mathcal{H}(D)$. Therefore, by Lemma $1,(s, o) \in \mathcal{H}(D)$ and hence, by the definition of $\mathcal{I}, u \in \mathcal{I}(D)$. Also, from the definition of $D$, it can be shown that $\{s \frown u \mid u \in \mathcal{I}(D)\} \subseteq \mathcal{I}(C)$. Therefore,

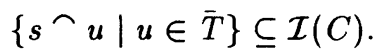

\section{CASE STUDY}

To demonstrate the differences between the finite and the infinite trace models, we develop a management system for process identifiers (PIDs). This system is implemented using a bus where all numbers are transmitted serially. As the number of PIDs is not bounded (a PID can be any natural number) and any free PID can be chosen by the system, the number of communications on the bus - a local operation - is unbounded but finite. This causes the system to diverge in the finite trace model but not in the infinite trace model.

A connection diagram of the system can be found in Fig. 1. Every box in the diagram stands for an Object$\mathrm{Z}$ class. The semantics of the combination is defined by CSP parallel composition and hiding (see below).

The type $P I D$ is just an abbreviation for a natural number $(P I D==\mathbb{N})$. The class $P I D$ man has two operations: A request from the environment for new PIDs by

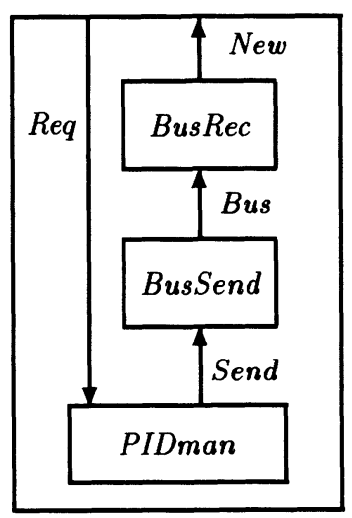

Figure 1 Connection Diagram for PIDbus $R e q$ is answered with the operation Send that transfers a new PID to the bus. Releasing PIDs that are no longer used is not considered in this simple example.

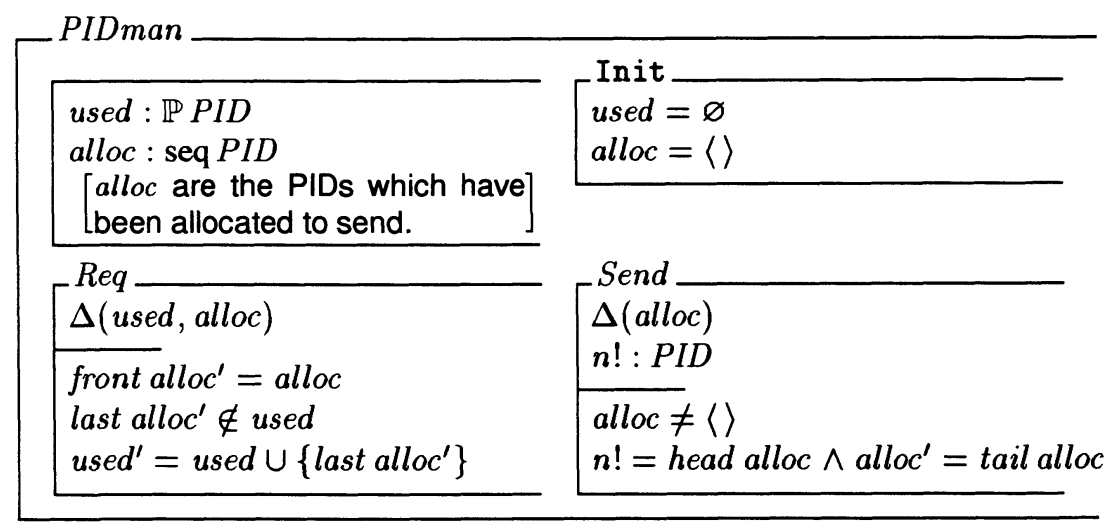

The output of the PID manager is transmitted via a serial bus to the environment. The type $S T A T U S$ is used to code the control flow of the bus and the type $S B I T$ extends a bit with the stop signal $S$ denoting end of transmission.

$$
\text { STATUS ::= trans | wait }
$$




\section{$S B I T::=0|1| S$}

The status trans holds during transmission of bits over the bus. When the system is ready to receive a new $P I D$ the status wait holds. The class BusSend receives a natural number and sends it bitwise over the bus.

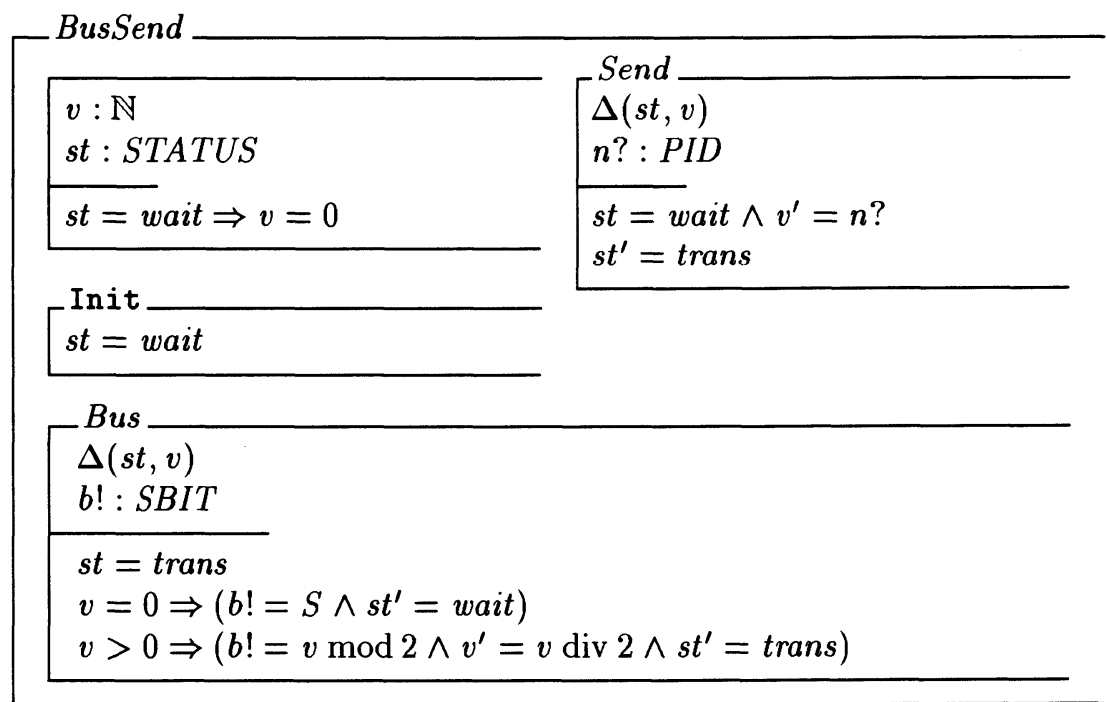

The class BusRec receives bits over the bus, decodes the bits to a number and sends the result to the environment. The variable $b c$ counts the number of bits already received.

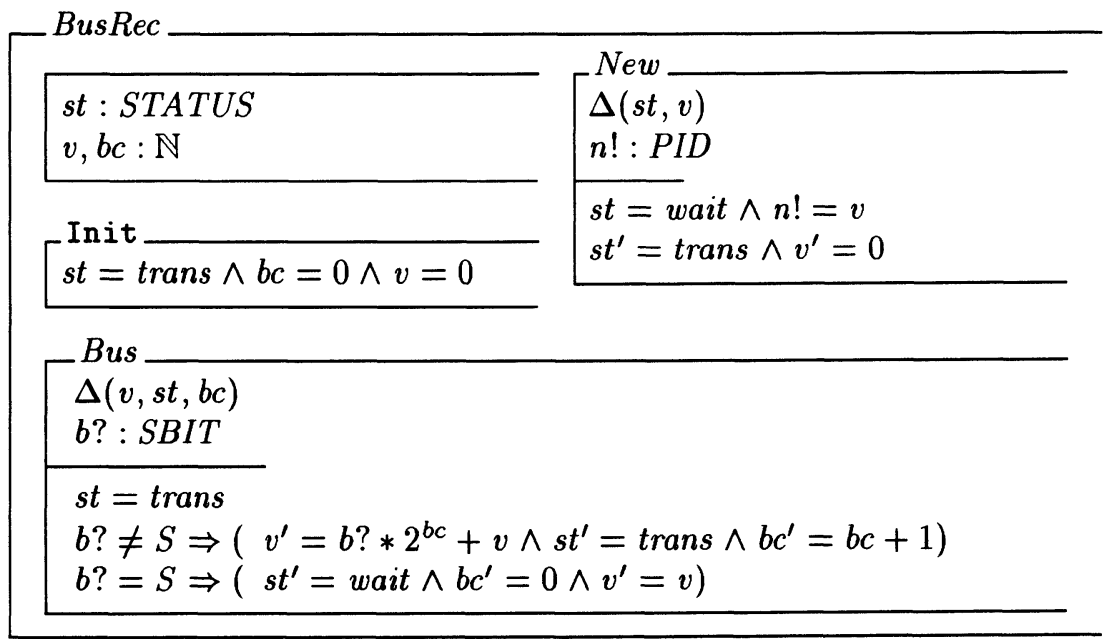

The system PIDbus is defined by the following CSP expression (compare Fig. 1): PIDbus $=\left(\right.$ PIDman $\|_{\{\mid \text {Send } \mid\}}$ BusSend $\|_{\{|B u s|\}}$ BusRec $) \backslash\{\mid$ Send, Bus $\mid\}$ 
where $C_{1} \|_{X} C_{2}$ denotes the CSP parallel composition of $C_{1}$ and $C_{2}$ synchronising on the set of events $X$. The notation $\{\mid$ Send $\mid\}=\{$ Send.n $\mid n \in P I D\}$ abbreviates the set of events of the operation Send.

By the CSP hiding operator \, the operations Send and Bus are made invisible to the environment. These operations are called local operations. The different semantics of hiding in the CSP models let PIDbus diverge in the finite trace model, but not in the infinite trace model:

The divergences of the hiding operator in the finite trace model are:*

$$
\begin{aligned}
\mathcal{D}(C \backslash X)= & \left\{(s \backslash X)^{\frown} t \mid s \in \mathcal{D}(C)\right\} \cup \\
& \left\{s \frown t \mid \forall n \bullet \exists s_{n}: \mathcal{T}(C) \bullet s_{n} \backslash X=s\right\}
\end{aligned}
$$

where $\mathcal{T}(C)=\{s \mid(s, \varnothing) \in \mathcal{F}(C)\}$ is the set of traces of $C$. Divergent traces are a result of finite traces on the local operation with no upper bound. There are at least $n$ div $2+2$ operations Bus if the PID $n$ is transmitted. As there is no upper bound for PIDs, there is no upper bound for the number of local operations of PIDbus. Thus PIDbus diverges after receiving a request for new PIDs.

A divergent trace in the infinite trace model is a result of an infinite trace involving only local operations.

$$
\mathcal{D}(C \backslash X)=\left\{(u \backslash X)^{\frown} t \mid u \in \mathcal{I}(C) \wedge u \backslash X \text { is finite }\right\} \cup\{s \backslash X \mid s \in \mathcal{D}(C)\}
$$

As we argued above, there is no infinite trace of Bus in PIDbus. As well, the other local operation, Send, can only occur once during a transmission of a PID. Thus PIDbus is not divergent in the infinite trace model.

\section{REFINEMENT}

In this section we investigate the differences in the refinement rules for both semantic models.

CSP refinement is based on set inclusion: A process $P$ refines a process $Q$ in the finite trace model if $P$ is more deterministic $\mathcal{F}(P) \subseteq \mathcal{F}(Q)$ and less divergent $\mathcal{D}(P) \subseteq \mathcal{D}(Q)$. We write $Q \sqsubseteq_{F} P$ if $Q$ is refined by $P$ in the finite trace model.

For the infinite trace model, the additional condition $\mathcal{I}(P) \subseteq \mathcal{I}(Q)$ must hold. We write $Q \sqsubseteq_{I} P$ to denote this refinement. The symbol $\sqsubseteq$ denotes refinement in both models.

Refinement of Object- $Z$ classes can be defined similar to $Z$ data refinement. A class $C$ refines a class $A$ (both having the same set of operations), if every step of $C$ can somehow be simulated by the class $A$. The exact definition relies on a retrieve relation Retr that relates the states of $C$ and $A$. Thus Retr can be written in the form

*Note that in [18] a refined version of the hiding operator is given that can cope with some forms of unbounded nondeterminism. But this definition is not widespread in the CSP literature and as the main problem remains, we use the simpler standard definition for hiding in a finite trace model. 
[A.State; $C$.State $\mid P]$ for some predicate $P$. (A.State refers to the state schema of class $A$. Similar notation is used to refer to the other schemas of a class.) We assume in this section that $C$ and $A$ do not have variables with the same name.

The class $C$ is a forward simulation of the class $A$ if the following holds for all operations $o p$.

1. The initial states are related: $C$.Init $\Rightarrow \exists A$.Init $\bullet$ Retr

2. The preconditions of related states are equivalent: $\operatorname{Retr} \Rightarrow$ (pre $A . o p \Leftrightarrow \operatorname{pre} C . o p$ ) where pre $o p=\exists$ State $^{\prime} \bullet o p$ is the precondition of an operation.*

3. The state change of $C . o p$ can be simulated by the operation $A . o p$ for corresponding states: $\operatorname{Retr} \wedge C . o p \Rightarrow \exists A$. State $^{\prime} \bullet \operatorname{Retr}^{\prime} \wedge A . o p$

The definition of backward simulation is similar. It is omitted here.

The relation between these simulation techniques and CSP refinement was worked out by He [14], Josephs [17], Woodcock and Morgan [26] and Butler [4]. They prove that forward simulation is sound, i.e. if $C$ is a simulation of $A$ then $A \sqsubseteq C$ holds. This is proven by Hallerstede [13] in the finite trace model for a combination of CSP and $\mathrm{Z}$ similar to our combination of Object-Z and CSP. This proof is easily extended to the infinite trace model.

Differences occur, however, if the simulation technique is extended to local operations which are not visible for the environment. Thus we investigate extensions of the simulation rules to prove $A \sqsubseteq C \backslash X$ where $X$ is the set of events of the local operations $L$. Simulation rules for this situation are called weak simulation [6].

\subsection{Weak simulation in the infinite trace model}

The idea of a weak simulation rule is that the abstract state is not changed by a local operation in the concrete class.

Let $C$ and $A$ be classes and Retr a retrieve relation and $L$ the set of local operations of $C$. Then $C$ is a weak forward simulation of $A$ if the following holds for all operations op of $A$ :

1. The initial states are related: $C$.Init $\Rightarrow \exists A$.Init $\bullet$ Retr

2. The preconditions of related states are equivalent if no local operation is enabled.

$$
(\operatorname{Retr} \wedge \forall l: L \bullet \neg \text { pre } C . l) \Rightarrow(\text { pre } A . o p \Leftrightarrow \operatorname{pre} C . o p)
$$

Note that refusals cannot be observed if a local operation is enabled. Therefore the equivalence of the preconditions must only hold if no local operation is enabled.

3. The state change of $C . o p$ can be simulated by the operation $A . o p$ for corresponding states: Retr $\wedge C . o p \Rightarrow \exists A$. State $^{\prime} \bullet$ Retr $^{\prime} \wedge A . o p$

and there exists a schema term $\cong$ [C.State; $\left.t: \mathbb{N} \mid P_{\text {term }}\right]$ such that term is defined for all states of $C$ and for all local operations $l \in L$ the following holds:

4. $l$ does not change the abstract state:

$C . l \wedge$ Retr $\Rightarrow \exists A$. State' $\bullet \Xi A$. State $\wedge$ Retr $^{\prime}$

5. $l$ does not diverge: $C . l \wedge$ term $\Rightarrow t^{\prime}<t \wedge$ term $^{\prime}$

*Note that the definition of pre here is different to that in $\mathrm{Z}$ where the outputs are also hidden. 


\subsection{Weak simulation in the finite trace model}

For the finite trace model we have to add an extra condition concerning bounded nondeterminism. Increase of the value $t$ of the schema term must be finitely bounded.

Hence, $C$ is a weak forward simulation of $A$ if 1.-5. from above and the following extra condition holds

6. There exists a constant $b: \mathbb{N}$ that bounds the increase of $t$ for all operations $c$ :

$$
\text { C.c } \wedge \Delta t e r m \Rightarrow t^{\prime}<t+b
$$

Furthermore the initial value of $t$ must be bound: $C$. Init $\wedge$ term $\Rightarrow t<b$ A refined version of this rule that also takes the communicated parameters into account can be found in [10].

\subsection{Case study}

We use the example from Section 5 to indicate the application of the simulation rules. We have designed class PIDman such that it is a specification of the complete system PIDbus when Send is renamed to New, i.e. we want to prove PIDman[New/Send $] \sqsubseteq_{I}$ PIDbus.

To apply the weak simulation rule, we have to remove the parallel composition in the definition of PIDbus. This can be done by a rule for parallel composition from [10]: Parallel composition of classes with disjoint state spaces is basically equivalent to conjunction. Thus CSP parallel composition can be expressed by Object- $Z$ inheritance. According to this rule, the parallel composition of PIDman, BusSend and BusRec is equivalent to the following class that inherits all three classes.

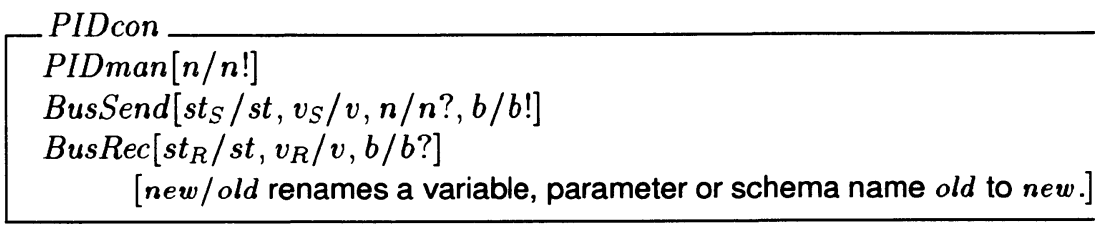

Note that the common named schemas of the inherited classes are implicitly conjoined.

With proper renaming, we can apply the rule for weak forward simulation for the infinite trace model to prove

$$
\text { PIDman }\left[\text { New/Send }, \text { used }_{A} / \text { used }_{\text {, alloc }} / \text { alloc }\right] \sqsubseteq_{I} \text { PIDcon } \backslash\{\mid \text { Send, Bus } \mid\}
$$

using the schemas retr and term from Fig. 2. A proof of the side conditions will be published in a technical report. As we expect, the proof of the extra side condition 6 in the finite case fails; there is no bound for the variable $t$ after the operation Send.

Note that the case study, although very simple, shows a general principle of using Object- $Z$ and CSP for the development of communicating systems: The class PIDman corresponds to the specification of some layer $N$ that is implemented us- 


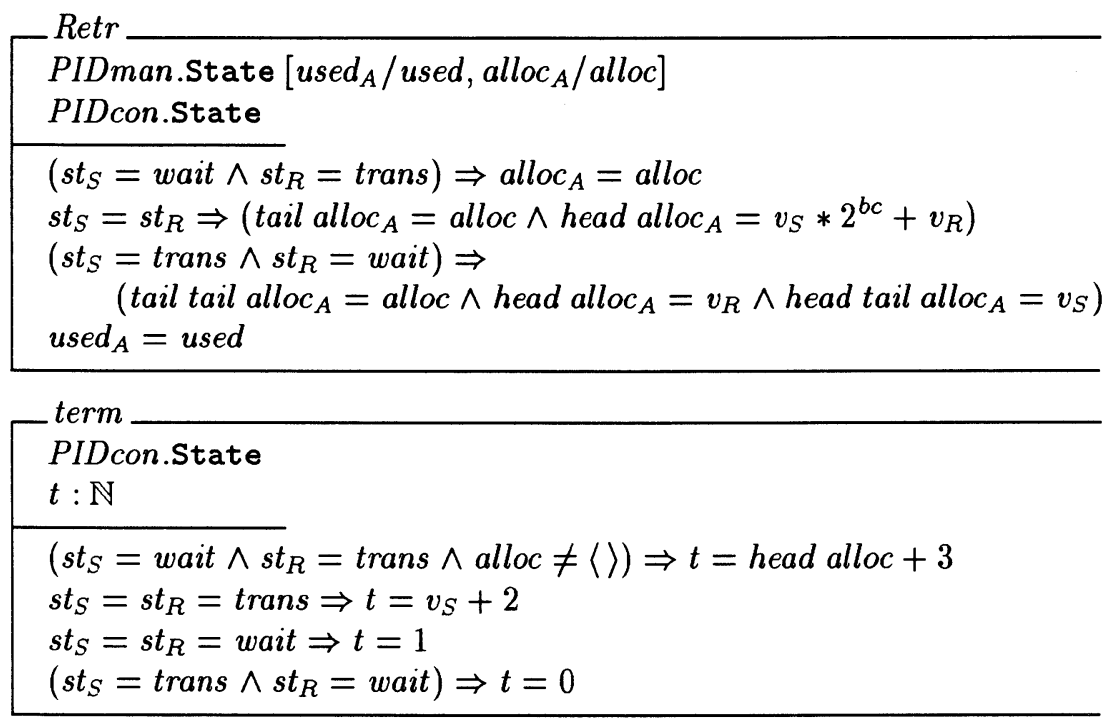

Figure 2 Retrieve Relation and Termination Schema for the Case Study

ing the functionality of some lower layer $N+1$, the bus. The two steps in this section, parallel composition and weak simulation, are generally applicable in this situation.

\section{SPECIFYING FAIRNESS}

In this section, we investigate the specification of fairness properties as a possible application of the infinite traces model. Fairness properties state that an operation which is either repeatedly or continuously enabled must eventually occur. They therefore affect only the infinite, and not the finite, traces of a process.

Consider specifying a buffer which never does an infinite sequence of inputs without outputting. Such a specification may be modelling a finite buffer in which case, an implementation would need to restrict the behaviour so that an input cannot be performed when the capacity of the buffer is reached. Alternatively, it may be modelling a finite buffer which is shared by a number of other clients and whose capacity, therefore, from the point of view of a particular client, changes nondeterministically.

In standard CSP, such fairness properties can be captured by the introduction of an auxiliary "supervising" process but not at a higher level of abstraction. In Object$\mathrm{Z}$, they can be captured by the use of temporal logic predicates to restrict the set of histories of a class. For example, the buffer described above could be specified as follows.

Buffer ${ }_{1}[T]$ Buffer $[T]$ 
The class $B$ uffer $r_{1}$ inherits $B$ uffer from Section 2. Inheritance can be resolved here by replacing $B u f f e r$ with its definition. The history predicate $\square \diamond(O u t$ occurs) (always eventually Out occurs) restricts the infinite histories of $B u f f e r_{1}$ to those histories which do not end in an infinite sequence of In operations. (See [21] for details.)

However, we cannot specify such fairness properties when the class is going to be used in the CSP setting because axiom 8 does not hold. Buffer ${ }_{1}$ must do any finite sequence of $I n$, but it cannot do an infinite trace of $I n$.

Fairness properties can only be placed on events which are totally under the control of a process and not its environment [1]. Therefore, to specify a fair buffer, we need to model the decision to perform an Out operation as internal to the buffer. For example, the class $B$ uffer $r_{2}$ has an auxiliary variable next_out modelling the buffer's decision to perform an Out operation next. It can only do this when there is an item in the buffer to output.

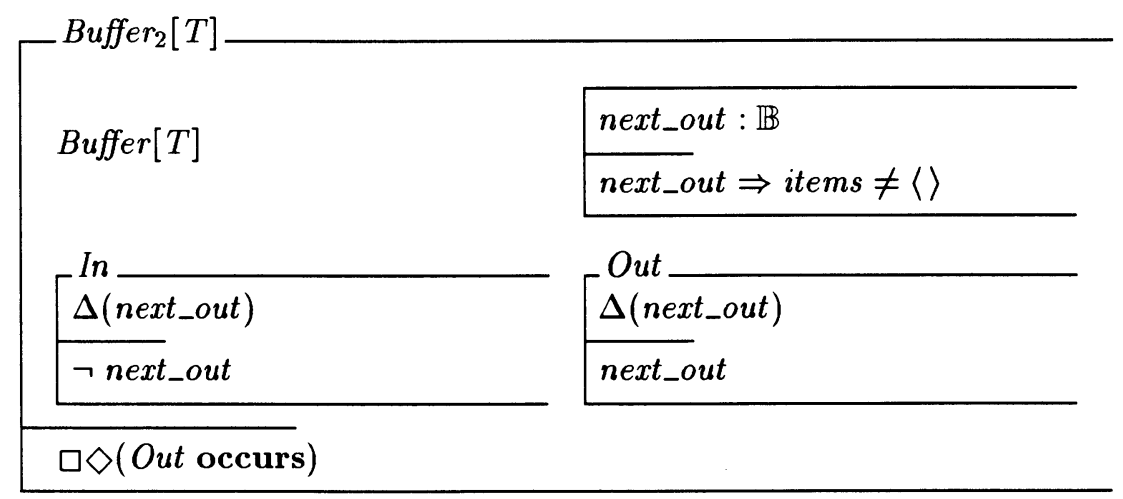

In this case, the fairness condition on Out makes sense. If we place $B$ uffer $r_{2}$ in an environment which continuously offers events corresponding to the operation In but never events corresponding to the operation Out, it will eventually deadlock (when next_out becomes true). Note that the resulting finite trace with only events corresponding to $I n$ is a possible trace of the class. The fairness property only restricts the class' infinite traces. Axiom 8 holds for Buffer $_{2}$ (although the proof from section 4.3 does not translate directly for this situation).

An other form of operations that are totally under control of a process are local operations. However, fairness constraints on local operations can implicitly put constraints on visible operations and thus making axiom 8 invalid.

The infinite trace model can, therefore, be used as a means to specify fairness properties abstractly in a combined Object-Z/CSP notation. But appropriate restrictions are required to prevent the occurrence of classes which do not fulfill all axioms of the CSP model. The exact form of such restrictions and the practicalities of enforcing them requires further investigation.

\section{CONCLUSION}

In this paper we have investigated the influence of the finite and the infinite trace models on the combination of Object-Z and CSP. First of all, the underlying idea of 
deriving a CSP semantics from the histories semantics of an Object- $\mathrm{Z}$ class and the proof of the necessary axioms can be carried out without difficulty in both models. In fact both CSP semantics for Object- $Z$ are equivalent if only bounded nondeterminism is considered.

From a theoretical point of view, the infinite trace model seems to be preferable: It can handle all forms of unbounded nondeterminism properly, the rules for weak simulation have fewer side conditions and, thus, are more flexible. It is even possible to handle fairness constraints under certain restrictions; although the exact formulation of the restrictions is a topic for further research.

However, the finite trace model is more widespread in the CSP community. Tools like the CSP model checker FDR [12] or an encoding of CSP in Isabelle [25] are only available for the finite trace model. Although the refinement rule is more complicated, the simulation technique is only complete ${ }^{*}$ in the finite trace model $[4,13]$. Furthermore, it is not clear whether the restricted use of fairness constraints in the infinite trace model is a significant advantage since the necessity of modelling internal choice in Object- $Z$ classes can often complicate specifications. This is evident from the example in Section 7.

To decide which model is preferable, the role of unbounded nondeterminism in the application domain of the combination of Object-Z and CSP has to be considered. If unbounded finite sequences of events occur, the infinite trace model is the better choice. However, in real systems such unbounded sequences of events do not occur. For example, there would be a bound for PIDs in any real system. If we used the type $P I D==0 \ldots \max P I D$ (for some natural number $\max P I D$ ) instead of $\mathbb{N}$ in the case study of Section 5, we could safely use the finite trace model. The specification would still be fairly abstract as we wouldn't have to specify the exact value of $\max P I D$. Further case studies will show if such restrictions can be found in general.

\section{Acknowledgements}

The work of C. Fischer is part of the project UniForM supported by the German Ministry for Education and Research (BMBF) under grant No. FKZ 01 IS 521 B2. G. Smith is supported by a research fellowship granted by the Alexander von Humboldt-Stiftung, Germany.

\section{REFERENCES}

[1] M. Abadi and L. Lamport. Composing specifications. In J.W. de Bakker, W.-P. de Roever, and G. Rozenberg, editors, REX Workshop, volume 430 of Lecture Notes in Computer Science, pages 1-41. Springer-Verlag, 1990.

[2] T. Bolognesi and E. Brinksma. Introduction to the ISO specification language LOTOS. Computer Networks and ISDN Systems, 14(1):25-59, 1988.

[3] S.D. Brookes and A.W. Roscoe. An improved failures model for communicating processes. In Pittsburgh Symposium on Concurrency, volume 197 of Lecture Notes in Computer Science, pages 281-305. Springer-Verlag, 1985.

[4] M. J. Butler. A CSP Approach To Action Systems. PhD thesis, University of Oxford, 1992.

[5] E. Cusack and G.H.B. Rafsanjani. ZEST. In Object-Orientation in Z, Workshops in Computing, pages 113-126. Springer-Verlag, 1992.

\footnotetext{
* This means that every relation $A \subseteq C$ for classes $A$ and $C$ can be proven by successive application of
} forward and backwards simulation. 
[6] J. Derrick, E. Boiten, H. Bowman, and M. Steen. Weak refinement in Z. In J. Bowen and M. Hinchey, editors, ZUM'97: The Z Formal Specification Notation, volume 1212 of LNCS, pages 369-388, 1997.

[7] J. Derrick, E.A.Boiten, H. Bowman, and M. Steen. Supporting ODP - translating LOTOS to Z. In First IFIP International workshop on Formal Methods for Open Object-based Distributed Systems (FMOODS). Chapman \& Hall, 1996.

[8] J. Dong, R. Duke, and G. Rose. An object-oriented approach to the semantics of programming languages. In G. Gupta, editor, 17th Annual Computer Science Conference (ACSC'17), pages 767-775, 1994.

[9] R. Duke, G. Rose, and G. Smith. Object-Z: A specification language advocated for the description of standards. Computer Standards and Interfaces, 17:511-533, 1995.

[10] C. Fischer. Combining CSP and Z. Technical report, University of Oldenburg, 1997.

[11] C. Fischer. CSP-OZ: A combination of Object-Z and CSP. To appear in Formal Methods for Open Object-Based Distributed Systems (FMOODS '97), 1997.

[12] Formal Systems (Europe) Ltd. Failures-Divergence Refinement: FDR 2, Dec 1995. Preliminary Manual.

[13] S. Hallerstede. Die semantische Fundierung von CSP-Z. Master's thesis, University of Oldenburg, 1997. In German.

[14] J. He. Process simulation and refinement. Formal Aspects of Computing, 1(3):229-241, 1989.

[15] C.A.R. Hoare. Communicating Sequential Processes. International Series in Computer Science. Prentice-Hall, 1985.

[16] ITU Recommendation X.901-904. Open Distributed Processing - Reference Model Parts 1-4, July 1995.

[17] M.B. Josephs. A state-based approach to communicating processes. Distributed Computing, 3:9-18, 1988.

[18] A.W. Roscoe. An alternative order for the failures model. Journal of Logic and Computation, 3(2), 1993.

[19] A.W. Roscoe. Unbounded nondeterminism in CSP. Journal of Logic and Computation, 3(2), 1993.

[20] A.W. Roscoe and G. Barrett. Unbounded nondeterminism in CSP. In Mathematical Foundations of Programming Semantics, volume 442 of Lecture Notes in Computer Science, pages 160-193. Springer-Verlag, 1989.

[21] G. Smith. An Object-Oriented Approach to Formal Specification. $\mathrm{PhD}$ thesis, Department of Computer Science, University of Queensland, 1992.

[22] G. Smith. A fully abstract semantics of classes for Object-Z. Formal Aspects of Computing, 7(3):289-313, 1995.

[23] G. Smith. A semantic integration of Object-Z and CSP for the specification of concurrent systems. To appear in Formal Methods Europe (FME '97), 1997.

[24] J.M. Spivey. The Z Notation: A Reference Manual (2nd Ed.). International Series in Computer Science. Prentice-Hall, 1992.

[25] H. Tej and B. Wolff. A corrected failure-divergence-model for CSP in Isabelle/HOL. To appear in Formal Methods Europe (FME '97).

[26] J. C. P. Woodcock and C. C. Morgan. Refinement of state-based concurrent systems. In Proceedings of VDM Symposium 1990, volume 428 of LNCS, pages 340-351. SpringerVerlag, 1990. 